\title{
Hongrie : un dispositif innovant
}

\section{Théa Reves}

\section{(2) OpenEdition}

\section{Journals}

Édition électronique

URL : http://journals.openedition.org/ries/3975

DOI : 10.4000/ries.3975

ISSN : 2261-4265

\section{Éditeur}

Centre international d'études pédagogiques

\section{Édition imprimée}

Date de publication : 7 septembre 1995

Pagination : 119-129

ISSN : 1254-4590

\section{Référence électronique}

Théa Reves, «Hongrie : un dispositif innovant », Revue internationale d'éducation de Sèvres [En ligne], 07 | 1995, mis en ligne le, consulté le 19 avril 2019. URL : http://journals.openedition.org/ries/3975 ; DOI : 10.4000/ries.3975

Ce document a été généré automatiquement le 19 avril 2019

(c) Tous droits réservés 


\title{
Hongrie : un dispositif innovant
}

\author{
Théa Reves
}

1 L'objectif pédagogique du lycée français bilingue de Budapest, hautement innovant, est de former de nouvelles générations de professionnels dotés d'une instruction de qualité qui soient en mesure de maîtriser la langue française presque comme une langue maternelle. Ce que propose le lycée français bilingue correspond pleinement aux programmes d'immersion en langue étrangère récemment introduits dans le système éducatif hongrois. La raison d'être implicite de ces programmes est que les élèves soient en mesure, après avoir poursuivi des études universitaires plus poussées, d'occuper des positions de responsabilité au niveau socioéconomique, comblant ainsi les besoins d'une société nouvelle, libérale, tournée vers le monde. L'objectif déclaré de ces programmes d'immersion hongrois n'est pas de préparer de futurs linguistes professionnels, des professeurs de langues ou des interprètes, mais des cadres spécialisés, qui rempliront des fonctions dans divers domaines de la vie professionnelle.

2 Cette étude de cas présente le programme d'immersion réalisé au lycée français bilingue de Budapest.

\section{Programmes d'immersion}

3 Les programmes d'immersion hongrois qui débutèrent dans les années 1980 doivent être considérés dans le contexte socioculturel complexe de ce pays. Au début des années 1980, à l'aube d'un changement général au niveau politique, social et économique dans la vie de la Hongrie, une relative démocratisation de l'éducation s'est mise en place, à la recherche d'un équilibre délicat entre traditionalisme dogmatique et modernisme pragmatique dans l'éducation, les institutions éducatives devant acquérir davantage d'autonomie professionnelle.

4 Sur le modèle de certaines institutions bilingues d'avant la Seconde Guerre mondiale en Hongrie (comme le Reichsdeutsches Gymnasium ou le Liceo Italiano) et en cherchant à imiter les programmes d'immersion occidentaux, tout en profitant de l'expérience acquise des systèmes bilingues dans le bloc oriental (par exemple le système bulgare), les responsables hongrois initièrent un programme partiel expérimental, le lycée bilingue, 
en $1986^{1}$.L'importance de cette entreprise doit être envisagée dans le contexte presque totalement monolingue et uniculturel de ce pays².

$5 \quad$ La décision de développer des programmes d'immersion dans des langages occidentaux étrangers se révélait d'une importance culturelle particulière, dans la mesure où, depuis quarante ans, le russe avait été la seule langue obligatoire enseignée dans le système éducatif hongrois ; les langues étrangères occidentales n'avaient, au cours de ces quatre décennies, été enseignées que sporadiquement, surtout dans des institutions privées et des cours spéciaux de langue.

6 L'introduction de programmes de langues occidentales impliquait par conséquent la levée des barrières intellectuelles imposées en Hongrie au cours des quatre dernières décennies.

7 Ce projet soulevait également des difficultés au plan politique, puisqu'il impliquait un parrainage d'agences occidentales (comme la Banque mondiale, la fondation américaine Soros, etc.) ou d'institutions étrangères d'enseignement des langues (par exemple: l'Institut français, le British Council, l'Institut Goethe), donc l'introduction d'idéologies étrangères, de professeurs pour lesquels les langues étrangères à enseigner soient la langue maternelle et du matériel étranger. En outre, un risque d'« hémorragie des cerveaux » était à craindre parmi les futurs diplômés de ces établissements bilingues ${ }^{3}$.

Néanmoins, en 1987, un enseignement bilingue débutait dans quinze institutions à travers tout le pays; les langues choisies furent l'anglais, le français, l'allemand, l'italien et l'espagnol. Tout en se référant aux expériences occidentales, les programmes d'immersion organisés dans le bloc oriental diffèrent profondément de leurs modèles. La première différence est liée à la dimension sociolinguistique de la langue d'immersion : alors que, dans la plupart des programmes-modèles occidentaux, la langue à acquérir est une langue seconde (L2) (celle de l'environnement), la langue-cible des programmes menés dans le bloc oriental est une langue étrangère, qui n'est pas utilisée dans l'environnement immédiat ou élargi de l'élève. Cette différence touche à l'efficacité de l'apprentissage de la langue à acquérir, dans la mesure où l'élève n'est pas exposé à cette langue en dehors de la salle de classe. Elle induit des effets sur la motivation. En effet, alors que l'acquisition d'une langue L2 est une nécessité évidente de survie et doit donc être réalisée à tout prix, la compétence dans une langue étrangère ne se révélera payante que par la suite, au moment d'embrasser une profession ou de bâtir une carrière.

9 La moyenne d'âge des élèves constitue une autre différence entre les programmes d'immersion dans les pays d'Europe de l'Est et la plupart de ceux du Canada et des ÉtatsUnis. Alors que la majeure partie des programmes d'immersion occidentaux «âge tendre » commence au jardin d'enfants et/ou à l'école élémentaire, ceux d'Europe de l'Est étaient menés pour la plupart dans des classes de lycée, commençant donc avec des adolescents de quatorze ans. Ce choix s'appuie sur des conclusions tirées de recherches ${ }^{4}$ suggérant que les élèves plus âgés jouissent d'un avantage marqué sur les plus jeunes, grâce à leurs compétences de lecture plus développées et leurs bases de connaissances, ainsi qu'à leurs capacités sémiotiques en général. En outre, étant donné que les aptitudes linguistiques sont interdépendantes, les élèves plus mûrs sont ceux qui maîtrisent le mieux les opérations mentales de transfert de leur compétence en langue principale vers la langue cible. Ce processus s'applique à toutes les capacités, à l'exception bien évidemment de la prononciation et de la facilité d'élocution. 
10 Le programme des sections bilingues, en Hongrie, s'étend sur cinq années au lieu de quatre dans le cursus standard. Cependant les lycéens se présentent au même examen de fin d'études secondaires. Les matières qui ont été enseignées en langue étrangère sont évaluées dans cette langue. La première année du programme (classe 0 ) est entièrement consacrée à l'apprentissage de la langue étrangère. Dès la seconde année, les matières enseignées dans une langue étrangère sont, dans la plupart des programmes d'immersion, les mathématiques, la physique, la chimie, la biologie, la géographie, l'histoire générale, voire les disciplines artistiques dans certains établissements. Le choix des matières dépend à l'évidence à la fois du type d'établissement, de la possibilité de recruter les enseignants possédant les connaissances et les qualifications appropriées tant dans la discipline enseignée que dans la langue à acquérir.

11 En 1995, plus de quarante lycées en Hongrie avaient introduit un programme bilingue partiel. Dans la plupart des cas, il s'agit de lycées d'enseignement général, bien que certaines écoles professionnelles, comme par exemple les écoles de tourisme et les écoles hôtelières, aient récemment, pour des raisons pratiques, introduit des programmes bilingues partiaux. Le nombre de disciplines enseignées dans la langue étrangère varie de deux à dix selon les besoins spécifiques et le type d'établissement.

\section{Le lycée français bilingue de Budapest}

12 L'un des trois lycées français bilingues de Hongrie, le seul situé dans la capitale, Budapest, a été l'objet d'étude et d'observation de septembre 1994 à janvier 1995. Par l'observation de classes, on s'efforça de saisir une image complète sous le plus grand nombre d'angles possible: niveau de compétence atteint, progrès effectués par les élèves; méthodes d'enseignement selon les disciplines; comportement verbal des élèves, dans les matières enseignées en L1 (hongrois) et en première langue étrangère (français) et dans les cours de seconde langue étrangère (anglais, allemand, italien, etc.).

13 L'étude prévoyait également des échanges avec les élèves, des entretiens avec le directeur du lycée, des professeurs, d'anciens élèves de l'établissement, des parents, des responsables du ministère, des enseignants de l'université et des responsables de la mise en œuvre de ces programmes bilingues. Les entretiens étaient menés en L1 ou en langue étrangère, au choix de l'interrogé ; ils étaient enregistrés, transcrits et traduits.

Parmi les questions proposées aux responsables ministériels, aux membres de l'université, au directeur et au corps enseignant, certaines concernaient les données de bases (contexte de l'initiation, premières difficultés, compétences des élèves à l'entrée, structure du programme, etc.), d'autres les comportements et attitudes (importance du projet, sentiment de réalisation, difficultés envisagées, etc.).

15 Les entretiens avec les élèves portaient sur les attitudes, les motivations, l'effet du cursus bilingue sur les projets d'avenir, etc. Les parents étaient interrogés sur leurs aspirations (réalisations universitaires de leurs enfants), leurs attitudes par rapport à la langue et plus particulièrement sur le rôle du français dans l'éducation de leurs enfants. Les anciens élèves avaient à s'exprimer, de façon rétrospective sur leurs attitudes et le bénéfice de ce programme bilingue, etc.

16 Les questionnaires concernant les acquis personnels, l'auto-évaluation et les attitudes furent proposés aux élèves de première année, ainsi qu'aux élèves de dernière année, avant l'examen final. Ils pouvaient être remplis de façon anonyme ou non, au choix de 
l'élève. Ils visaient à obtenir des informations sur les études antérieures de chacun, les attitudes envers la langue et la culture françaises, les raisons du choix d'un tel programme bilingue, le statut socio-économique des parents.

Des questionnaires d'auto-évaluation permettaient aux élèves de première et de cinquième année de juger par eux-mêmes de leur compétence orale et écrite en langue étrangère dans divers contextes de communication. Ces auto-évaluations étaient ensuite comparées à l'évaluation des enseignants. Les chercheurs disposaient aussi d'une documentation sur les programmes, les examens, ainsi que de comptes rendus du corps enseignant et des conseillers pédagogiques hongrois et étrangers.

\section{Présentation de la section bilingue}

18 Le programme français bilingue représente une des sections d'un grand lycée. Outre le programme bilingue sur cinq ans, l'établissement abrite également deux programmes de quatre ans, le cursus d'un lycée standard et un programme d'apprentissage intensif de langues étrangères. Ces diverses sections se trouvent sous la juridiction d'une seule et même administration. Le même corps enseignant y exerce partiellement.

Les financements viennent du ministère de l'éducation, via le conseil régional. Le coût par élève est de 50 \% supérieur à celui des programmes non bilingues. Ce surcroît est dû à la subdivision des élèves en deux ou trois groupes.

Le taux d'abandon en cours d'études est très réduit (5-8\%) par an. Les abandons se produisent pour l'essentiel à la fin de la classe 0 (première année), en raison des difficultés d'apprentissage du français langue étrangère. Le contrôle est exercé par les mêmes autorités que pour les établissements standards d'enseignement secondaire : le ministère de l'éducation pour les contenus éducatifs, et le conseil régional en ce qui concerne l'administration et les finances. L'établissement bénéficie, en plus, de conseils de la part d'institutions hongroises (l'institut pédagogique national, le département de littérature et de linguistique françaises de l'université de Budapest et le département de formation des professeurs de l'université). Des représentants d'organismes culturels français (service culturel français de l'ambassade, Instituts français et, à l'occasion, experts français) apportent un soutien pédagogique.

21 La section française bilingue semble avantagée par rapport aux autres sections bilingues. Elle est fortement appréciée et son statut est également reconnu officiellement par le ministère français de l'éducation nationale. Ses examens suffisent pour l'accès à l'université française. Des échanges scolaires sont organisés avec le soutien d'institutions éducatives et $\mathrm{du}$ gouvernement français. Les professeurs de français se voient régulièrement offrir des bourses pour des cours de perfectionnement ou des universités d'été en France; les professeurs des disciplines enseignées en français bénéficient régulièrement de cours de formation et de perfectionnement en France. Ce dispositif d'initiation concerne aussi les professeurs enseignant en hongrois dans les classes du programme bilingue. La bibliothèque, sans cesse enrichie de matériel didactique supplémentaire (dotation de livres de la part de l'Institut français et d'autres institutions françaises installées en Hongrie) est à la disposition de tout établissement.

Les élèves sont recrutés hors carte scolaire et peuvent se porter candidats à toute section bilingue. Certains élèves (34\%) assurent avoir choisi le lycée français bilingue pour des raisons utilitaires d'importance pratique de la langue française. La majorité (66\%) 
considère le programme français comme l'occasion de faire l'apprentissage d'une belle langue et de se familiariser avec une culture qui jouit d'un prestige incontesté en Europe. Le milieu socioculturel des élèves semble refléter les ambitions d'une société qui s'ouvre progressivement à de nouveaux horizons intellectuels. Dans la plupart des cas les deux parents exercent une profession libérale (pères : $48 \%$; mères : $42 \%$ ) ou sont employés de bureau (pères : $33 \%$; mères $45 \%$ ), parfois enfin sont cols bleus (pères : $18 \%$; mères : $12 \%$ ). Dans leur grande majorité, les élèves sont des filles ( $81 \%$ en première année, classe 0 , et $63 \%$ en cinquième année au terme du cursus).

\section{Le programme d'études}

Les objectifs du programme d'études sont d'enseigner le français en recréant l'environnement culturel le plus authentique possible. Par exemple, les textes littéraires et historiques sont analysés et évalués selon le modèle de l'explication de textes à la française. L'un des prérequis du lycée français bilingue est que seuls les lycéens dotés d'une maitrise très développée de leur langue peuvent suivre le programme intensif de français langue étrangère. Cette position repose sur l'hypothèse d'une interdépendance linguistique : si les capacités en L1 ne sont pas suffisamment développées, une exposition poussée en L2 ne stimulera pas le bilinguisme et risquera même d'entraver le développement en $\mathrm{L} 1$.

La première année du programme bilingue, ou classe 0 , est entièrement consacrée à l'apprentissage du français. Le français, en tant que langue étrangère, est enseigné neuf heures par semaine. Les classes sont divisées en trois groupes de dix à douze élèves. La terminologie propre aux disciplines qui seront l'objet du programme bilingue au cours des quatre années suivantes (mathématiques, physique, géographie, biologie, histoire) est enseignée onze heures par semaine. La classe est alors divisée en deux groupes de quinze à dix-huit élèves. Ce dédoublement se poursuit jusqu'à la dernière classe de lycée. Le programme est celui enseigné dans tous les lycées standards du pays. Seule la langue d'enseignement diffère. Une seconde langue étrangère, anglais, allemand, italien, russe ou espagnol est étudiée durant trois ans.

\section{La démarche pédagogique}

Les enseignants du lycée français bilingue se recrutent à la fois parmi des personnels de langue maternelle française, diplômés des universités de pays francophones, et parmi des enseignants de langue maternelle hongroise, diplômés d'universités du pays. La double compétence exigée explique le manque d'enseignants et l'initiative des universités qui ont mis en place récemment des programmes spéciaux associant disciplines et langue étrangère (exemple: histoire et français, biologie et anglais, etc.). Les enseignants hongrois qui enseignent en français se voient offrir des bourses d'études d'un an en France. Les élèves soulignent néanmoins que certains de leurs professeurs éprouvent des difficultés à enseigner leur discipline en français, étant donné qu'ils ne peuvent procéder avec autant de naturel que s'ils avaient appris tous les contenus en français.

Les manuels scolaires sont ceux utilisés dans les établissements standards. Ils sont traduits mot à mot du hongrois en français. Le programme national d'études peut donc être respecté. Cependant, ce matériel didactique est souvent démodé, voire obsolète. Il est le fruit de l'époque antérieure. Il doit donc être complété par des matériels appropriés 
(livrets d'accompagnement, documents visuels, supports pour un enseignement assisté par ordinateur). Il provient de dons de l'étranger (ministère de l'éducation nationale en France, au Canada, en Suisse, etc.). Il est proposé la plupart du temps sous sa forme originale, et quelquefois il fait l'objet d'une adaptation aux besoins hongrois.

L'apprentissage de la langue commence avec une approche strictement orale (répétitions, phrases modèles). Après deux semaines, le cours progresse vers un contenu plus communicatif. L'accent est alors mis sur la grammaire, de façon relativement conventionnelle avec un recours aux techniques de traduction, explications des règles en hongrois et d'exemples en français. Dans les classes suivantes l'explication de textes classiques littéraires prend une importance grandissante. Les élèves lisent et comprennent des passages littéraires plutôt difficiles, développent une approche critique et proposent des analyses argumentées. Les rédactions, dans ces deux dernières classes, sont lues à haute voix et même parfois suivies de débats.

En principe, après sélection, les nouveaux élèves sont admis dans le programme bilingue de leur choix. Les deux premières années de lancement du programme, quelques cinq cents élèves avaient demandé à être admis au lycée français bilingue ; ils durent passer un examen conçu par le ministère de l'éducation: épreuves écrites uniquement, questionnaires à choix multiples en mathématiques, compréhension écrite en hongrois et tests psychotechniques. $15 \%$ des candidats ont été retenus. Il semble aujourd'hui rétrospectivement que c'est la nouveauté de cet examen hautement centralisé qui a assuré son efficacité. Depuis, avec le temps, on assiste à une baisse de niveau. Aussi, il a été décidé de confier à chaque établissement bilingue le soin de préparer son propre examen d'entrée. Ce nouvel examen est nettement moins formel. Il prend en compte la personnalité des candidats au travers d'un plus grand nombre d'épreuves. Il est plus exigeant en matière de raisonnement logique, de créativité. Il vérifie le niveau de maîtrise en langue maternelle. Le lycée français bilingue propose depuis peu une alternative, un cours de trois jours en été, permettant d'apprécier le comportement et l'aptitude intellectuelle des postulants.

\section{Les compétences des élèves}

Plusieurs programmes d'évaluation ont été réalisés pour étudier les bénéfices éducatifs et le rapport coût/efficacités.

Au lycée français bilingue, des expérimentations/contrôles sont régulièrement conduites. Pour vérifier le niveau de compétence en français, des groupes témoins d'élèves appartenant aux classes standards sont sollicités. De même, pour juger des progrès dans les disciplines enseignées en français, une évaluation formative est proposée en parallèle dans les classes non bilingues de l'établissement. Les résultats indiquent que les performances des élèves du programme français bilingue sont, dans la plupart des cas, supérieures à celles des élèves des autres programmes bilingues, et bien au-dessus de celles des élèves de sections standards.

31 À leur sortie du lycée français bilingue, les élèves sont acceptés presque sans exception dans les universités les plus fermées en Hongrie comme à l'étranger.

À la demande de l'administration, aucun test formel n'est conduit dans les classes. Des entretiens structurés et des conversations personnelles sont menés avec divers groupes d'élèves. Ils apportent des indications sur leurs progrès et leurs performances. Les 
entretiens avec des élèves de classe 0 , après les quatre premiers mois d'études, ont révélé un démarrage très rapide. Les compétences orales des élèves, la fluidité et la rigueur, semblent justifier pleinement l'idée de consacrer toute une année à l'apprentissage exclusif d'une langue. Pour ce qui est de la terminologie, les observations faites permettent d'apprécier l'aisance des élèves dans le choix non contraint des mots. Les entretiens menés avec la classe terminale sont révélateurs du haut degré de compétence atteint en français. Cette compétence est très proche de celle maîtrisée en hongrois.

Des questionnaires d'auto-évaluation ont été utilisés après un an et demi d'études du français, et après quatre ans et demi. Les élèves devaient évaluer par eux-mêmes leur aptitude à accomplir en français un certain nombre de tâches. Ils devaient ensuite comparer leur aptitude à exécuter les tâches en français et en langue étrangère. Les résultats ont montré que les élèves évaluaient leur compétence orale (écoute et prise de paroles) $20 \%$ au-dessus de leur compétence écrite (lecture/écriture).

Ces appréciations furent comparées aux notes données par les professeurs de français. On a observé une corrélation élevée, pondérée cependant par le fait que l'évaluation par les élèves de leurs aptitudes orales se situait $15 \%$ au-dessus des notes attribuées et que les compétences écrites avaient été sous-estimées de $20 \%$ par les élèves. Ces discordances sont probablement la conséquence des méthodes surannées en usage où l'accent est davantage mis sur le travail écrit et formel des élèves. Ces supports et la pédagogie induite ont des effets grossissants.

\section{Un label de qualité}

Bien que les programmes bilingues anglais et allemands constituent, pour des raisons de succès professionnel immédiat dans un monde régi par la technologie, un choix populaire chez les candidats lycéens et leurs familles en Hongrie, le choix du français semble motivé par des ambitions plus hautes, intellectuelles, élitistes. La préférence pour un programme d'immersion français est apparemment inspirée par l'estime dont la langue et la culture jouissent dans l'opinion, ainsi que par le rôle joué par la France dans la Communauté européenne.

Dans les questionnaires d'attitude, les élèves de première année du lycée français bilingue ont été interrogés sur les raisons de leur choix. Seuls $34 \%$ ont mentionné des raisons utilitaires ; quelque $66 \%$ ont justifié leur choix par des raisons culturelles. Dans les classes plus élevées, les élèves semblaient avoir des motivations plus utilitaires lorsqu'on leur demandait de dresser une liste de bénéfices induits par leur futur diplôme: $39 \%$ prenaient en compte l'avantage instrumental (exercice d'une profession) ; $36 \%$ voyaient dans la maîtrise de cette langue étrangère un atout pour intégrer un système économique européen nouvellement organisé ; $70 \%$ pensaient que la connaissance du français les aiderait à se familiariser avec le monde occidental ; $33 \%$ prenaient en compte les voyages à l'étranger et seule une minorité ( $18 \%$ ) donnait des arguments plus généraux, comme la beauté de la langue française ou son importance dans la civilisation mondiale.

Bien que les entretiens informels durant les récréations et en classe aient été introduits en hongrois, certains élèves choisirent le français comme langue d'échange. Certains élèves se félicitèrent même de pouvoir avoir ainsi une conversation en français. Les motivations scolaires, l'attachement à l'école ont semblé être plutôt élevés, sans doute de par le sentiment d'appartenance à une institution prestigieuse et par le fait qu'une 
attention toute personnelle est accordée à chacun. Le soutien des parents joue également un rôle.

Des entretiens avec les anciens élèves ont montré un enthousiasme pour le programme et leur fierté d'avoir participé à ce projet. Des anciens élèves ont assuré qu'être diplômés du lycée bilingue français de Budapest leur avait conféré un statut spécial dans leurs études universitaires.

Les enseignants du programme français bilingue se sont montrés très motivés, en dépit de salaires extrêmement bas. Le programme prestigieux de cet établissement attire les meilleurs professeurs de français langue étrangère du pays; réussir à y exercer est reconnu comme une preuve d'excellence. Ceux dont le français est la langue maternelle apprécient très vivement les efforts accomplis pour diffuser la langue et les valeurs culturelles de la France. Ils semblent être devenus partie prenante de l'établissement, sincèrement attachés à leurs collègues et aux élèves et remplissant leur tâche avec loyauté et fiabilité. Les enseignants hongrois sont des passionnés de la langue et de la culture française. Certains d'entre eux expriment leurs attitudes dans des termes enthousiastes. Ils travaillent avec une remarquable constance et la source de leur motivation réside dans leur forte identification à l'établissement et aux objectifs du programme bilingue.

40 L'importance culturelle et plus particulièrement éducative des programmes d'immersion dans la Hongrie d'aujourd'hui peut être pleinement comprise si nous songeons à l'isolement linguistique et culturel dont a souffert la Hongrie pendant plusieurs générations. La distance linguistique entre le hongrois et les autres langues romanes ou germaniques d'Europe, l'ethnocentrisme en raison duquel la Hongrie fut maintenue à l'écart ont été la cause et l'effet, durant des siècles, de l'aliénation du pays. De plus, la Hongrie n'est sortie que récemment d'une époque d'assujettissement politique et de domination culturelle. Cet isolement a vraisemblablement contribué à créer une situation de faiblesse face aux progrès rapides de la communauté mondiale.

41 Conséquences naturelles, le manque d'intérêt pour les langues occidentales et les méthodes d'enseignement obsolètes ont caractérisé le système éducatif hongrois. Les langues modernes ont été enseignées comme des «langues mortes». Les méthodes de traduction et de grammaire ne visaient pas à encourager la communication ou l'enrichissement culturel. Le caractère innovant de cette immersion linguistique est de provoquer une approche vivante, de créer un cadre quasi naturel, authentique, pour l'acquisition d'une langue étrangère. C'est là sans nul doute, malgré les combats d'arrièregarde, une tendance qui s'impose dans l'enseignement des langues.

C'est pourquoi, dans le contexte socioculturel de la Hongrie au cours des années 1980, l'initiation et l'introduction à grande échelle d'une éducation bilingue et biculturelle fondées sur des valeurs culturelles occidentales a revêtu une importance considérable : ce fut une entreprise remarquable de la part d'un système éducatif aux prises avec le débat sur tradition et modernité. Dans la société hongroise monolingue, la seule langue étrangère enseignée pendant des siècles fut l'allemand, langue parlée de façon occasionnelle, surtout dans les zones urbaines de la partie ouest du pays. Dans l'empire des Habsbourg, dont faisait partie la Hongrie, parler français et connaître la littérature française étaient le privilège d'un petit secteur intellectuel d'une classe sociale économiquement privilégiée, souhaitant rivaliser avec une certaine aristocratie. Ces aspects socioculturels conjugués à l'héritage culturel, assurent à la langue française un prestige dont aucune autre langue européenne ne jouit en Hongrie. 
43 À en juger par les résultats de ses huit premières années d'existence, le lycée bilingue français de Budapest peut à juste titre s'enorgueillir de son succès éducatif, scolaire et social. Il serait à l'évidence de toute première importance de mener des projets de recherche empirique contrôlés et corroborés au plan statistique, ainsi que des études ethnographiques en profondeur, afin de vérifier et d'enrichir les résultats suggérés par cette première évaluation descriptive.

\section{NOTES}

1. A. Vamos, A ketnyelvu gimnaziumok helyenek es helyzetenek nehany kerdese, Unpublished report, 1986-1990. A. Vamos, «Melyik nyelven tudja (jobban) ? Egy ketnyelvu gimnaziumi felmeres tapasztalatai », Magyar Tudomany, 12, 1992, p. 1448-1455.

2. 98,5\% de la population parlent le hongrois comme langue maternelle ; 15,5\% déclarent parler ou écrire au moins une langue étrangère dont 6,41 \% le français, (Office central des statistiques, 1992).

3. P.A. Duff, «Innovations in Foreign Language Education: an Evaluation of Three HungarianEnglish Dual- Language Programs », Journal of Multilingual and Multicural Development, 12, 1991, p. $459-476$

P. Medgyes, «The National L2 Curriculum in Hungary ", Annual Review of Applied Linguistics, 13, 1993, p. 24-36

4. M. Swain, S. Lapkin, « Canadian Immersion and Adult Second Language Learning: What's the Connection?", Modern Language Journal, 73, 1989, p. 150-159.

K. R. Johnson, M. Swain, «From Core to Content: Bridging the L2 Proficiency Gap in Late Immersion ", Language in Education, 1994.

5. A. Vamos, op.cit.

INDEX

Index géographique : Hongrie

Mots-clés : enseignement bilingue

\section{AUTEUR}

THÉA REVES

Département d'anglais, université Bar-Ilan, Ramat-Gan, Israël 